\title{
Modelling and trading the gasoline crack spread: A non-linear story
}

\author{
Christian L. Dunis, Jason Laws and Ben Evans* \\ ${ }^{*}$ CIBEF — Centre for International Banking, Economics and Finance, JMU, John \\ Foster Building, 98 Mount Pleasant, Liverpool, L35UZ. E-mail: B.Evans@Ljmu.ac.uk
}

Received: 14th November, 2005

Christian Dunis is Professor of Banking and Finance at Liverpool John Moores University and Director of CIBEF.

Jason Laws is a lecturer and course leader of MSc International Banking and Finance at Liverpool John Moores University.

Ben Evans is an associate researcher with CIBEF and is currently working on his $\mathrm{PhD}$.

\section{Practical applications}

The research below has three main practical applications.

Firstly, the ability to predict WTI-GAS spread displays obvious advantages to oil refiners, whose profit maximisation depends on knowing when to buy and sell stocks of either WTI crude or unleaded gasoline. Refining when the WTI-GAS spread is large will lead to higher operating profits than refining when the WTI-GAS spread is small.

Secondly, the tests of non-linear cointegration show whether upside and downside moves are significantly different. In this case this was interpreted as the ability of major oil companies to control the price of unleaded gasoline. This method could be used for other spreads indicative of profit margins such as the soybean crush spread (the difference between soybeans and soybean products), spark spread (the difference between natural gas and electicity) or the frac spread (the difference between natural gas and propane).

Finally, from the perspective of a futures trader, the ability to predict the direction of the WTI-GAS spread using a simple fair value model as described here could be easily implemented as a comparatively low risk trading strategy. Further it is also demonstrated that the use of filters can enhance the risk/return profile of this strategy.
Abstract
This paper investigates the gasoline crack spread time series, using the non-linear cointegration method developed by Enders and
Granger (1998, 'Unit-root Tests and Asymmetric Adjustment with an Example
Using The Term Structure of Interest Rates', Journal of Business and Economic

Derivatives Use, Trading \& Regulation, Vol. 12 No. 1/2, 2006 pp. 126-145

(c) Palgrave Macmillan Ltd Ltd $1747-4426 / 06 \$ 30.00$ 
Statistics, Vol. 19, pp. 166-176). The spread can be viewed as the profit margin gained by cracking crude oil, and therefore any non-linearity can be interpreted in the context of the effect on market participants. Further, a number of non-linear neural networks are used to forecast the gasoline crack spread. The architectures used are multilayer perceptron, recurrent neural networks and higher order neural networks, these are benchmarked against a fair value non-linear cointegration model. The final models are judged in terms of out-of-sample annualised return and drawdown, with and without a number of trading filters. The results show, first, that the spread does indeed exhibit asymmetric adjustment, with movements away from fair value being nearly three times larger on the downside than on the upside. Secondly, the best trading model of the spread is the higher order neural network with the threshold filter, owing to a superior out-of-sample risk/return profile.

\section{INTRODUCTION}

The motivation for this paper emanates from events in the oil markets c. 2005. Price rises in the level of crude oil cause increasing prices of crude oil products, such as unleaded gasoline. This slows down growth and hurts the economy. ${ }^{1,2}$ In contrast many oil-refining companies have listed some of the largest profits in history. ${ }^{3,4}$

Further motivation for this research is the non-linear analysis of the gasoline crack spread. The ability to identify non-linearities in a time series can clearly influence the decision of which models should be chosen to forecast the time series accurately, and also the type of trading filter that should be employed.

This case seems to be ideal for the investigation of any non-linearity in the spread time series and, furthermore, whether such asymmetries are biased in favour or against oil refining companies. The question of whether the consumer of unleaded gasoline has been getting a 'fair deal' seems valid. Discovering (or otherwise) any non-linearity in the spread can answer this question.

The gasoline crack spread can be interpreted as the profit margin gained by processing crude oil into unleaded gasoline. It is simply the monetary difference between West Texas Intermediate crude oil and Unleaded Gasoline, both of which are traded on the New York Mercantile Exchange (NYMEX). The spread is calculated as shown in equation (1)

$$
S_{t}=G A S_{t}-W T I_{t}
$$

where $S_{t}$ is the price of the spread at time $t$ (in \$ per barrel), GASt is the price of unleaded gasoline at time $t$ (in $\$$ per barrel), and $W T I_{t}$ is the price of West Texas Intermediate crude oil at time $t$ (in $\$$ per barrel).

A few large oil companies govern the pricing relationship between crude oil and unleaded gasoline. These companies are some of the largest in the world and, of these companies, BP, Shell and Exxon Mobil dominate the market. It is possible, therefore, for these companies to act in their own interests and not necessarily in the interests of the end user.

The spread time series for the in-sample 
Figure 1: Gasoline crack spread price 1st January, 1995-25th April, 2003

GAS-WTI Spread

(0101/1995 - 25/04/2003)

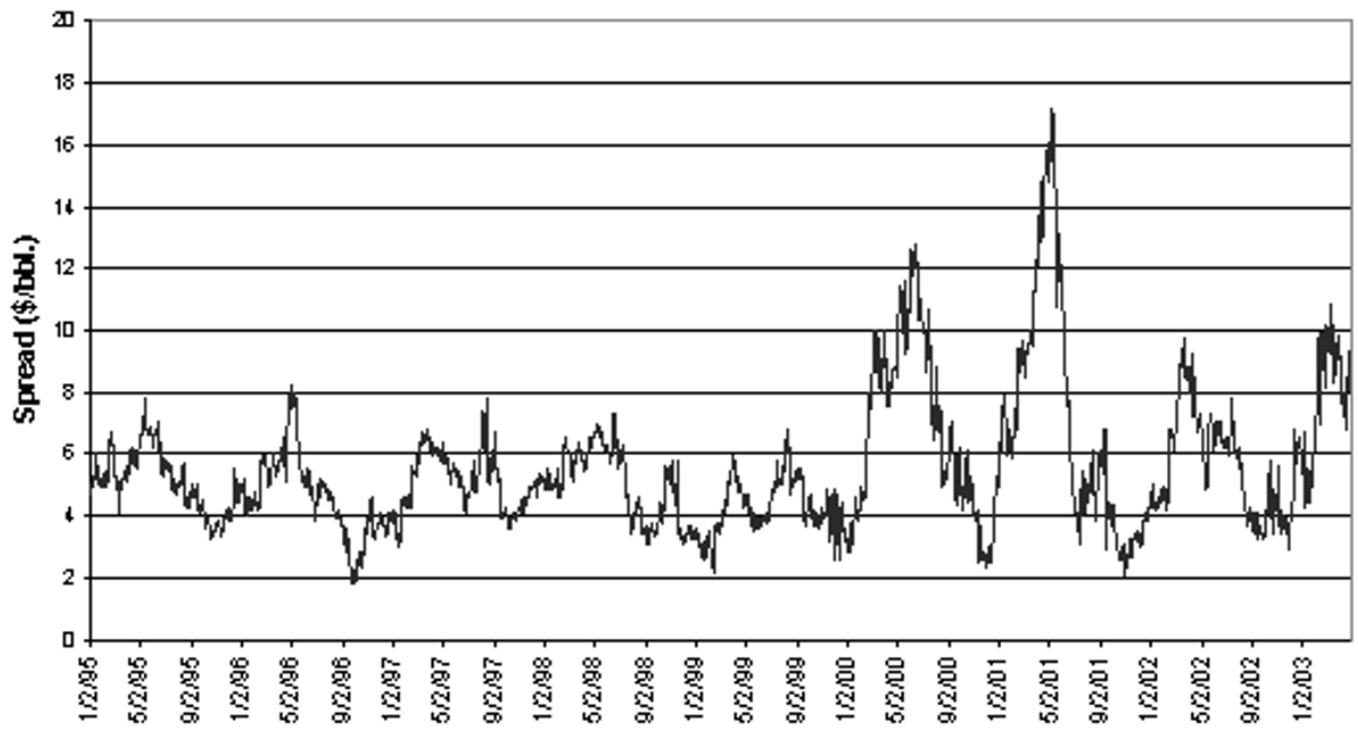

Date

period (1st January, 1995-25th April, 2003) is shown in Figure 1.

It is evident from Figure 1 that the spread shows mean reversion around approximately $\$ 5$ per barrel, but it is also evident that the spread shows asymmetry, with seemingly larger moves occurring on the upside of the long-term 'fair value' than on the downside. ${ }^{5}$ This could be a reason to expect the presence of non-linear cointegration, which is further explained in the fourth section of this paper.

This mean reversion is due to the fact that the spread is representative of a profit margin (the margin for refining crude oil into unleaded gasoline). The most common refining ratio for crude oil is $5: 3: 2$, that is 5 parts crude oil to 3 parts unleaded gasoline and 2 parts heating oil. This refining ratio means that crude oil products other than unleaded gasoline, particularly heating oil, can play significant roles in defining the pricing relationship between crude oil and unleaded gasoline. In addition, the range of products of crude oil is large and varied (gasoline, liquefied petroleum gas, naphtha, kerosene, gasoil and fuel oil, to name just the fuels). Fluctuating demand for these products can cause the gasoline crack spread to move away from the long-term fair value.

With such an unusual relationship, both economically and physically, the possibility exists that the movements of the spread are 
asymmetric. With this in mind, the hypothesis that the relationship between crude oil and unleaded gasoline exhibits non-linear adjustment is tested. It is a widely held view that, because of their market domination, adjustments in favour of oil refiners should be larger than adjustments that cause decreases in the refining margin. This is explained more fully below.

A fair value model is developed along the same lines as that of Evans et al. ${ }^{6}$ This is used as a benchmark for other non-linear models such as multi-layer perceptron (MLP), recurrent neural networks (RNN) and higher order neural networks (HONN). The models are used to forecast $\Delta S_{t}$, the daily change in the spread.

Finally, the correlation filter of Evans et al. ${ }^{6}$ is investigated and benchmarked against a more traditional threshold filter and, if the cointegration exhibits asymmetry, an asymmetric threshold filter. The exact specifications of these filters are included in the fifth section of this paper.

This paper is set out as follows: the next section details some of the relevant literature; the third section explains the data and methodology; the fourth section defines the trading models used; the fifth section defines the filters that have been employed; and the sixth and final sections give the results and conclusions, respectively.

\section{LITERATURE REVIEW}

Cointegration was first introduced by Engle and Granger. ${ }^{7}$ The technique is to test the null hypothesis that any combination of two series contains a unit root. If the null hypothesis is refuted and the conclusion is that a unit root does not exist, the combination of the two series is cointegrated. This is explained further below. As explained in the previous section, the spread may exhibit larger moves in one direction than in the other, this is known as asymmetry. Since the traditional unit root test has only one parameter for the autoregressive estimate, it assumes upside and downside moves to be identical or symmetric.

Non-linear cointegration was first introduced by Enders and Granger, ${ }^{8}$ who extended the unit root test by considering upside and downside moves separately, thus allowing for the possibility of asymmetric adjustment. The exact specification of this model is shown below.

This technique has been employed by, among others, Enders and Dibooglu, ${ }^{9}$ who test purchasing power parity (PPP) and find 'cointegration with threshold adjustment holds for a number of European countries on a bilateral basis'. Further, they conclude that 'central banks attempt to influence certain types of exchange rate movements and not others would seem to be a prima facie case against any type of symmetric adjustment'.

Boucher $^{10}$ investigates rational bubbles in the US and French stock markets, concluding that 'conventional cointegration tests fail to detect a long-run relationship between stock prices, dividends and proxies of the risk premium for US sample period 1953:1-2003:2, while the asymmetric cointegration tests uncover them'.

The trading of cointegrated time series is investigated by Evans et al., ${ }^{6}$ who develop a 
simple fair value model, as described below. They use this model to trade the WTI-Brent spread, finding that the fair value model competes very well against some more sophisticated models owing to the low level of trading activity and, therefore, transaction costs.

With the increasing processing power of computers, rule-induced trading has become far easier to implement and test. Kaastra and Boyd ${ }^{11}$ investigate the use of neural networks (NN) for forecasting financial and economic time series. They conclude that the large amount of data needed to develop working forecasting models involved too much trial and error. In contrast, Chen et al. ${ }^{12}$ study the 30-year US Treasury bond using a NN approach. The results prove to be good, with an average buy prediction accuracy of 67 per cent and an average annualised return on investment of 17.3 per cent.

Krishnaswamy et al. ${ }^{13}$ attempt to show the development of $\mathrm{NN}$ as modelling tools for finance. In turn, they cite valuable contributions from Kryzanowski et al. ${ }^{14}$, Refenes et al. ${ }^{15}$, Bansal and Viswanathan ${ }^{16}$ and $\mathrm{Zrilli}^{17}$ in the field of stock market and individual stock prediction, proving that not only do NN outperform linear regression models, but that NN are 'superior in dealing with structurally unstable relationships, notably stock market returns'. ${ }^{13}$ This research kick-started the search for increasingly more advanced NN architectures.

Recurrent networks or Elman networks were first developed by Elman ${ }^{18}$ and possess a form of error feedback, which is further explained in the subsection 'Recurrent neural network'. These networks are generally better than MLP networks, but they do suffer from long computational times. ${ }^{19}$ According to Saad et al., ${ }^{20}$ however, compared with other architectures this should not matter much: 'RNN has the capability to dynamically incorporate past experience due to internal recurrence, and it is the most powerful network of the three in this respect ... but its minor disadvantage is the implementation complexity'. ${ }^{20}$

Higher order NN were first introduced by Giles and Maxwell ${ }^{21}$ and were called 'tensor networks', although the extent of their use in finance is limited. Knowles et al. ${ }^{22}$ show that, despite shorter computational times and limited input variables on the EUR/USD time series, 'the best HONN models show a profit increase over the MLP of around 8 per cent'. A significant advantage of HONN is detailed in Zhang et al.:23 'HONN models are able to provide some rationale for the simulations they produce and thus can be regarded as 'open box' rather than 'black box'. Moreover, HONN are able to simulate higher frequency, higher order non-linear data, and consequently provide superior simulations compared to those produced by ANN (Artificial NN)'.

In recent years, there has been an expansion in the use of computer trading techniques, which has once again called into doubt the efficiency of even very liquid financial markets. Lindemann et al. ${ }^{24}$ suggest that it is possible to achieve abnormal returns on the Morgan Stanley High Technology 35 index, using a Gaussian mixture NN model. Lindemann et al. ${ }^{25}$ justify the use of the same 
Table 1: In-sample and out-of-sample dates
Data set
Dates
No. of observations
In-sample
1st January, 1995-25th April, 2003
2,170
Out-of-sample
28th April, 2003-1st January, 2005
440

Table 2: Training and test period dates

$\begin{array}{llc}\text { Period of in-sample } & \text { Dates } & \text { No. of observations } \\ \text { Training } & \text { 1st January, 1995-17th August, 2001 } & 1,730 \\ \text { Test } & \text { 20th August, 2001-25th April, 2003 } & 440\end{array}$

model to trade the EUR/USD exchange rate successfully, an exchange rate noted for its liquidity.

This paper investigates the use of the fair value model as a trading tool in the gasoline crack spread market, but also as a benchmark against more state of the art models such as MLPNN, recurrent NN (RNN) and HONN, which are described more fully below.

\section{DATA AND METHODOLOGY}

\section{Data}

The dataset used is the daily closing price data of the NYMEX West Texas

Intermediate (WTI) for the crude oil and NYMEX Unleaded Gasoline (GAS). With both markets trading on the same exchange and closing at identical times, the problem of non-simultaneous pricing is avoided. Figure 1 shows the gasoline crack spread pricing series. The spread between the two pricing series is calculated as shown in equation (1).

The returns of this series are then calculated as follows:

$$
\begin{aligned}
\Delta S_{t}= & {\left[\frac{\left(G A S_{t}-G A S_{t-1}\right)}{\left(G A S_{t-1}\right.}\right.} \\
& \left.-\frac{\left(W T I_{t}-W T I_{t-1}\right)}{\left(W T I_{t-1}\right)}\right]
\end{aligned}
$$

where $\Delta S_{t}$ is the percentage return of spread at time $t$.

Forming the returns series in this way means that it is possible to present results with more conventional percentage return/risk profiles. This methodology was used by Butterworth and Holmes ${ }^{26}$ and Evans et al. ${ }^{6}$ to calculate percentage spread returns.

The dataset has been split into two sets: in sample and out of sample. They are shown in Table 1. 
In the case of the NN models, the in-sample dataset was further divided into two periods. They are shown in Table 2 .

The reason for the further segmentation of the in-sample dataset is to avoid overfitting. As described later in this paper, the networks are trained to fit the training dataset and stopped when returns on the test dataset are maximised.

\section{Rollovers}

Using non-continuous time series brings a unique problem, since any long-term study will require a continuous series. If a trader takes a position on a futures contract, which subsequently expires, he can take the same position on the next available contract. This is called rolling forward. The problem with rolling forward is that two contracts of different expiry but the same underlying may not (and invariably do not) have the same price. When the roll-forward technique is applied to a futures time series, it will cause the time series to exhibit periodic blips in the price of the contract. While the cost of carry (which actually causes the pricing differential) can be mathematically taken out of each contract, this does not leave us with a precisely tradable futures series.

As this study is dealing with futures spreads, both contracts have been rolled forward on the same day of each month (irrespective of the exact expiry dates). The cost of carry, which is the cause of the price difference between the cash and futures price is determined by the cost of buying the underlying in the cash market now and holding until the futures expiry. Since the cost of storage of both underlying is similar, they will approximately offset each other. We are left with a tradable time series with no cost of carry effect.

\section{Transactions costs}

In order to assess the returns of each model realistically, they are assessed in the presence of transactions costs. The transactions costs are calculated from an average of five bid-ask spreads on WTI and GAS (ten in total), taken from different times of the trading day. These are 0.094 per cent for WTI and 0.1004 per cent for GAS. Therefore, on the spread, there is a total round trip transaction cost of 0.1944 per cent. Since commission fees are generally small and varied, they have not been considered here.

\section{TRADING MODELS}

The following section contains descriptions of the trading rules employed. First, the non-linear fair value model is used to predict the value of $\Delta \mu_{t}$, the daily change in the cointegration equation residuals: This is used as a proxy for $\Delta \tilde{S}_{t}$, as the actual returns of the spread are very similar to the actual returns of the cointegration residuals, ${ }^{27}$ or $\Delta S_{t} \approx \Delta \mu_{t}$. This is then used as a benchmark to test the ability of three NN models to predict $\Delta S_{t}$. The use of this benchmark is justified by the obvious asymmetry of the time series, as is apparent from Figure 1.

\section{Non-linear cointegration}

Enders and Granger ${ }^{8}$ extend the Dickey-Fuller test ${ }^{28}$ to allow for the unit root hypothesis to be tested against an alternative of asymmetric adjustment. Here, 
this is developed from its simplest form; consider the standard Dickey-Fuller test

$$
\Delta \mu_{t}=\rho \mu_{t-1}+\varepsilon_{t}
$$

where $\varepsilon_{t}$ is a white noise process. The null hypothesis of $\rho=0$ is tested against the alternative of $\rho \neq 0 . \rho=0$ indicates that there is no unit root, and therefore $\mu_{i}$ is a stationary series. If the series $\mu_{i}$ are the residuals of a long-run cointegration relationship as indicated by Johansen, ${ }^{29}$ this simply results in a test of the validity of the cointegrating vector (the residuals of the cointegration equation should form a stationary series).

The extension provided by Enders and Granger $^{8}$ is to consider the upside and downside moves separately, thus allowing for the possibility of asymmetric adjustment. Following this approach leads to equation (4)

$$
\Delta \mu_{t}=I_{t} \rho_{1} \mu_{i-1}+\left(1-I_{t}\right) \rho_{2} \mu_{i-1}+\varepsilon_{t}
$$

where $I_{t}$ is the zero-one 'heaviside' indicator function. This paper uses the following specification

$$
I_{t}=\left\{\frac{1, i f \mu_{t-1} \geq 0}{0, i f \mu_{t-1}<0}\right.
$$

Enders and Granger ${ }^{8}$ refer to the model defined above as threshold autoregressive (TAR).

The null hypothesis of symmetric adjustment is $\left(H_{0}: \rho_{1}=\rho_{2}\right)$, which can be tested using the standard $F$-test (in this case the Wald test), with an additional requirement that both $\rho_{1}$ and $\rho_{2}$ do not equal zero. If $\rho_{1} \neq \rho_{2}$, cointegration between the underlying assets is non-linear. In this case a trading model as described in
Evans et al. ${ }^{6}$ will be misspecified and equation (4) should be used to predict $\Delta \mu_{i}$.

A fair value trading model has been developed from this procedure. In this model, equation (4) is used to estimate values of $\Delta \mu_{t}$ from values of $\mu_{t-1}$. The values of $\rho_{1}$ and $\rho_{2}$ are estimated for the in-sample period and fixed for the out-of-sample period, the actual values of $\rho_{1}$ and $\rho_{2}$ are shown in Table 3 .

\section{Multi-layer perceptron}

The reference NN model used in this paper is the MLP. The MLP network has three layers; they are the input layer (explanatory variables), the output layer (the model estimation of the time series) and the hidden layer. The number of nodes in the hidden layer defines the amount of complexity that the model can fit. The input and hidden layers also include a bias node (similar to the intercept for standard regression), which has a fixed value of 1 (see Lindemann et al. ${ }^{25}$ and Krishnaswamy et al). ${ }^{13}$

The network processes information as shown below:

(1) The input nodes contain the values of the explanatory variables (in this case lagged values of the change in the spread).

(2) These values are transmitted to the hidden layer as the weighted sum of its inputs.

(3) The hidden layer passes the information through a non-linear activation function and onto the output layer.

The connections between neurons for a 
Figure 2: A single output, fully connected MLP model

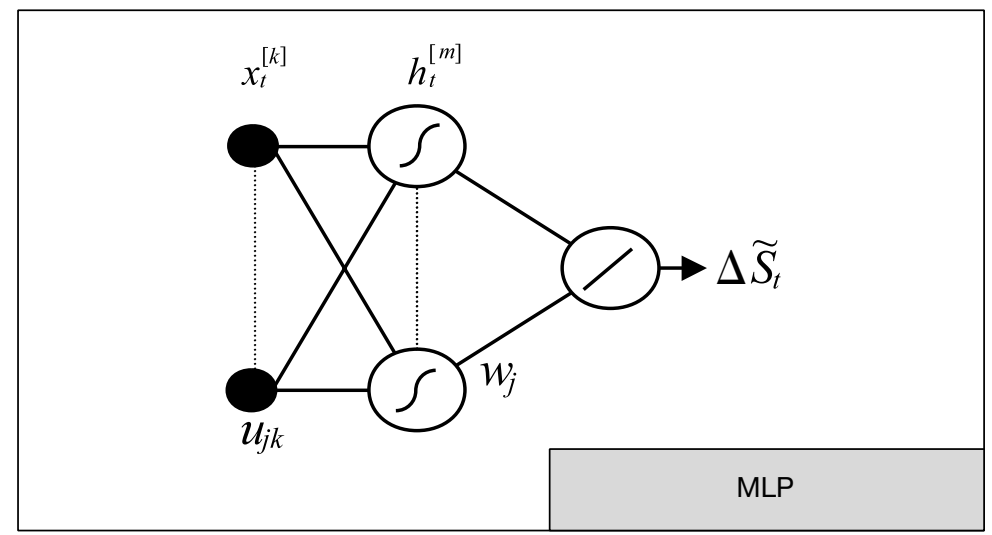

single neuron in the net are shown in Figure 2, where $x_{t}^{[n]}(n=1,2, ?, k+1)$ are the model inputs (including the input bias node) at time $t$ (in this case these are lags of the spread $) ; h_{t}^{[m]}(m=1,2, \ldots, m+1)$ are the hidden nodes outputs (including the hidden bias node); $\Delta \widetilde{S}_{t}$ is the MLP model output (predicted the percentage change in the spread at time $t) ; u_{j k}$ and $w_{j}$ are the network weights; (S) is the transfer sigmoid function $S(x)=1 / 1+\mathrm{e}^{-x} ; \oslash$ is a linear function $F(x)=\sum_{i} x_{i}$.

The error function to be minimised is

$$
E\left(u_{j k}, w_{j}\right)=\frac{1}{T} \sum\left[\Delta S_{t}-\Delta S_{t}\left(u_{j k}, w_{j}\right)\right]^{2}
$$

where $\Delta S_{t}$ is the target value (the actual percentage change in the spread at time $t$ ).

\section{Recurrent neural network}

While a complete explanation of the recurrent network is beyond the scope of this paper, a brief explanation of the significant differences between RNN and MLP architectures is presented below. For an exact specification of the recurrent network, see Elman. ${ }^{18}$

A simple recurrent network has activation feedback, which embodies short-term memory (see, for example, Elman ${ }^{18}$ ). The advantages of using recurrent networks over feedforward networks, for modelling non-linear time series, has been well documented in the past (see, for example, Adam et al. ${ }^{30}$ ). As described in Tenti, ${ }^{19}$ however, 'the main disadvantage of RNN is that they require substantially more connections, and more memory in simulation, than standard backpropagation networks', thus resulting in a substantial increase in computational time. Recurrent neural networks, however, can yield better results in comparison with simple MLPs owing to the additional memory input.

Connections of a simple recurrent network are shown in Figure 3.

The state/hidden layer is updated with external inputs, as in the simple MLP, but also with activation from previous forward propagation, shown as 'Previous State' in Figure 3. In short, the RNN architecture 
Figure 3: Architecture of Elman or RNN

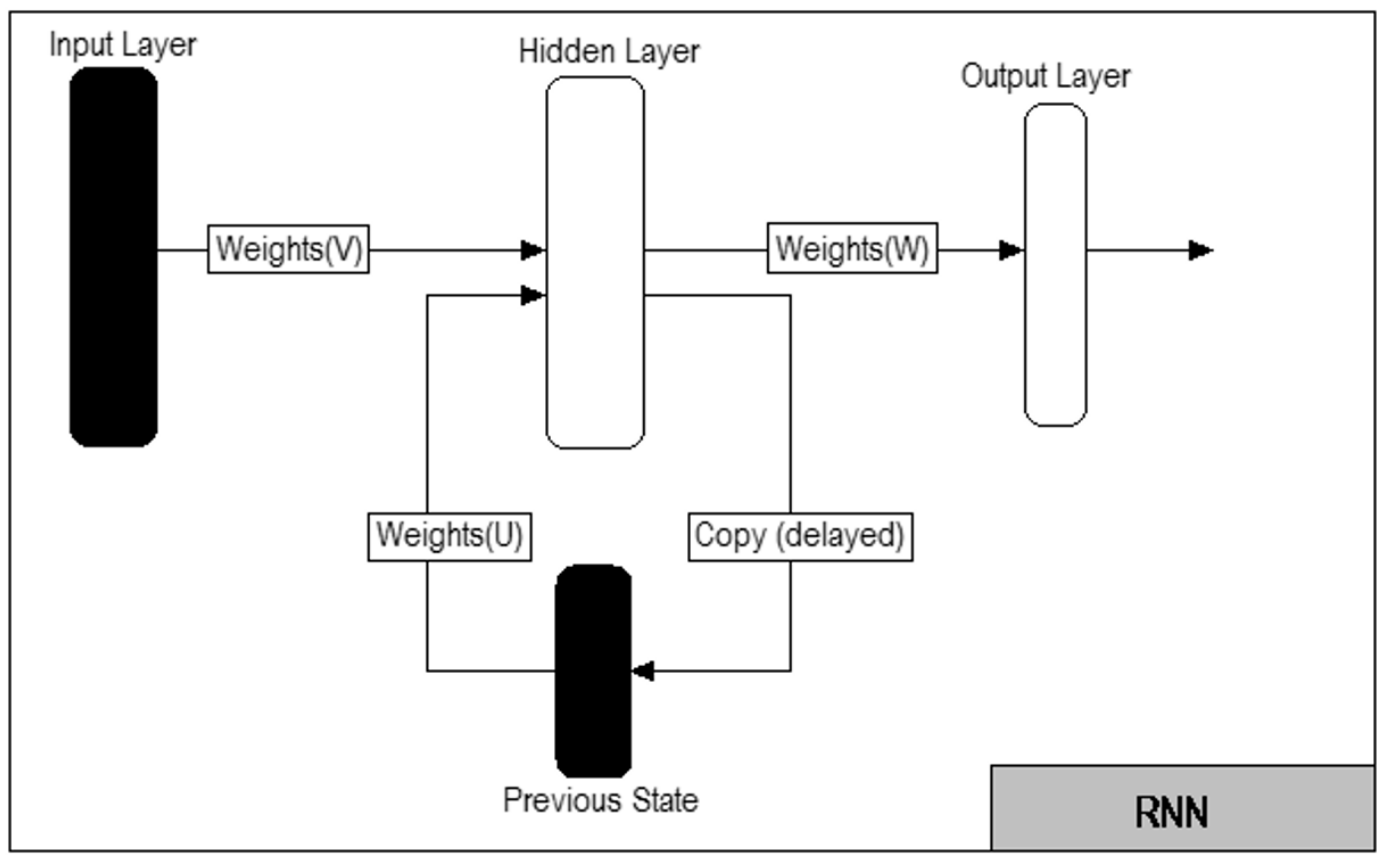

can provide more accurate outputs because the inputs are (potentially) taken from all previous values.

The Elman network in this study uses the transfer sigmoid function, error function and linear function, as described for the MLP architecture above. This has been done in order to be able to draw direct comparisons between the architectures of both models.

\section{Higher order neural network}

Higher order NN were first introduced by Giles and Maxwell, ${ }^{21}$ who referred to them as 'tensor networks'. While they have already experienced some success in the field of pattern recognition and associative recall, they have not been used extensively in financial applications. The architecture of a three input second-order HONN is shown in Figure 4:

Higher order NN use joint activation functions; this technique reduces the need to establish the relationships between inputs when training. Furthermore, this reduces the number of free weights and means that HONN can be faster to train than even MLPs. Because the number of inputs can be very large for higher order architectures, however, orders of 4 and over are rarely used.

Another advantage of the reduction of free weights means that the problems of overfitting and local optima affecting the results can be largely avoided. ${ }^{22}$ For a complete description of HONN, see Giles and Maxwell. ${ }^{21}$

The HONN in this study uses the 
Figure 4: Left, MLP with three inputs and two hidden nodes; right, second-order HONN with three inputs

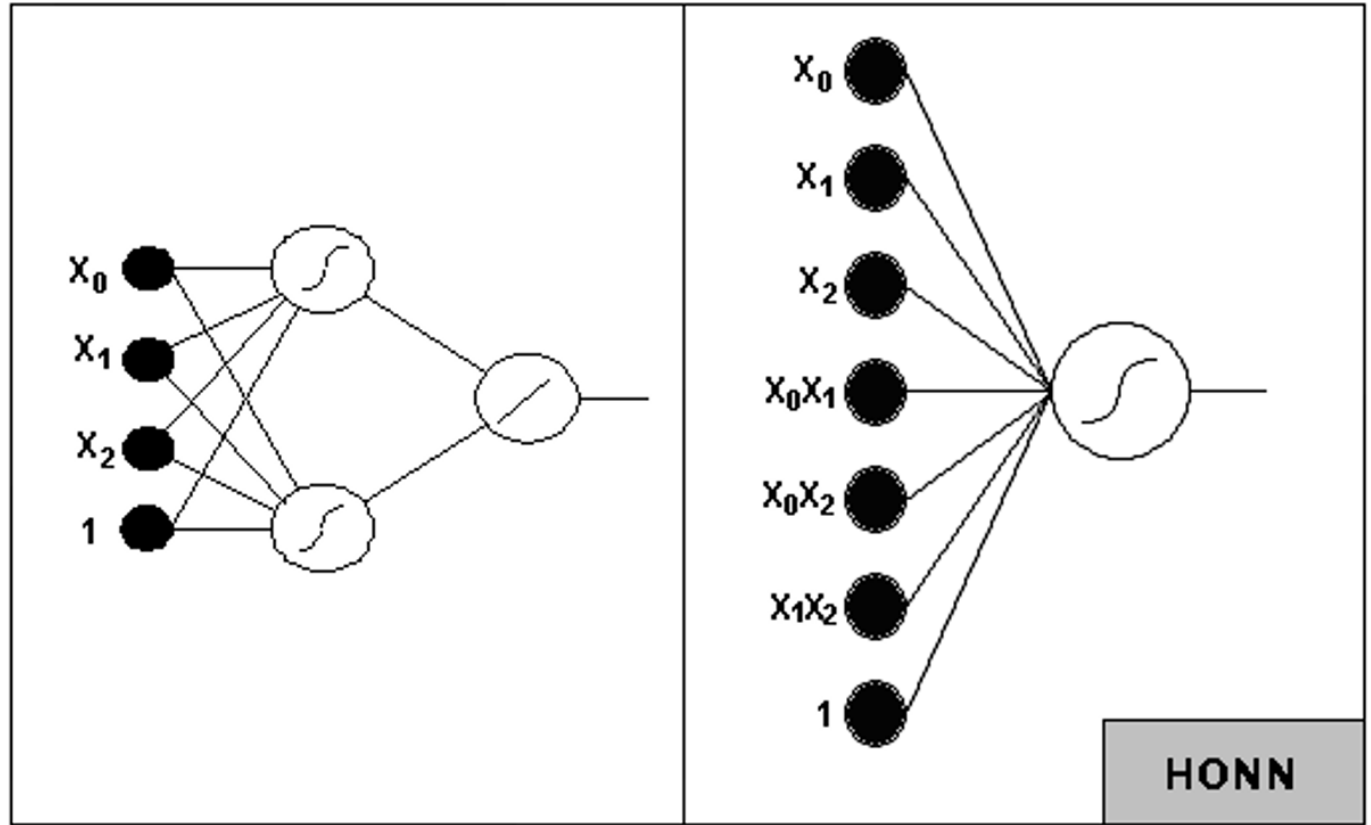

transfer sigmoid function, error function and linear function as described for the MLP architecture above. This has been done in order to be able to draw direct comparisons between the architectures of the models.

\section{Neural network training procedure}

The training of the network is of utmost importance, as it is possible for the network to learn the training subset exactly (commonly referred to as overfitting). For this reason, the network training must be stopped early. This is achieved by dividing the dataset into three different components (as shown in Table 2). First, a training subset is used to optimise the model, and the 'back propagation of errors' algorithm is used to establish optimal weights from the initial random weights. Secondly, a test subset is used to stop the training subset from being overfitted. Optimisation of the training subset is stopped when the test subset is at maximum positive return. These two subsets are the equivalent of the in-sample subset for the fair value model. This technique will prevent the model from overfitting the data, while also ensuring that any structure inherent in the spread is captured.

Finally, the out-of-sample subset is used to simulate future values of the time series, which for comparison is the same as the out-of-sample subset of the fair value model.

Since the starting point for each network 
is a set of random weights, a committee of ten networks has been used to arrive at a trading decision (the average estimate decides on the trading position taken). This helps to overcome the problem of local minima affecting the training procedure. The trading model predicts the change in the spread from one closing price to the next; therefore, the average result of all ten $\mathrm{NN}$ models was used as the forecast of the change in the spread or $\Delta S_{t}$.

This training procedure is identical for all the NN used in this study.

\section{TRADING FILTERS}

A number of filters have been employed to refine the trading rules, they are detailed in the following section.

\section{Threshold filter}

With all the models in this study, predicting the percentage change in the spread $\left(\Delta \tilde{S}_{t}\right)$, the threshold filter $X$ is as follows

If $\Delta S_{t}>X$ then go, or stay, long the spread, ${ }^{31}$

If $\Delta \tilde{S}_{t}<-X$ then go, or stay, short the spread, ${ }^{31}$

If $-X<\Delta \tilde{S}_{t}<X$, then stay out of the spread

where $\Delta \tilde{S}_{t}$ is the model's predicted spread return, and $X$ is the level of the filter (optimised in-sample).

With accurate predictions of the spread, it should be possible to filter out trades that are smaller than the level of the filter, thus improving the risk/return profile of the model.

\section{Asymmetric threshold filter}

As defined earlier, the relationship between WTI and GAS may not be the same, depending on whether the spread is above or below the fair value. With this in mind, an alternative filter is proposed: the asymmetric filter. The formalism is shown below:

If $\Delta \tilde{S}_{t}>\left|\boldsymbol{\rho}_{1}\right|^{\star} X$ then go, or stay, long the spread, ${ }^{31}$

If $\Delta \tilde{S}_{t}<-\left|\rho_{2}\right| \star X$ then go, or stay, short the spread, ${ }^{31}$

If $-\left|p_{2}\right| \star X<\Delta S_{t}<\left|\rho_{1}\right|^{\star} X$, then stay out of the spread,

where $\Delta \tilde{S}_{t}$ and $X$ are described above, and, the values of $\rho_{1}$ and $\rho_{2}$ are estimated in equation (4) over the in-sample period and fixed for the out-of-sample period.

\section{Correlation filter}

As well as the application of threshold and asymmetric threshold filters, the spreads were filtered in terms of correlation. The idea is to enable the trader to filter out periods of static spread movement (when the correlation between the underlying legs is increasing) and retain periods of dynamic spread movement (when the correlation of the underlying legs of the spread is decreasing). This was done in the following way.

A rolling $Z$-day correlation of the daily price changes of the two futures contracts is produced for the two legs of the spread. The $Y$-day change of this series is then calculated. From this a binary output of either 0 , if the change in the correlation is above $X$, or 1 , if the change in the 
Figure 5: Operation of the correlation filter

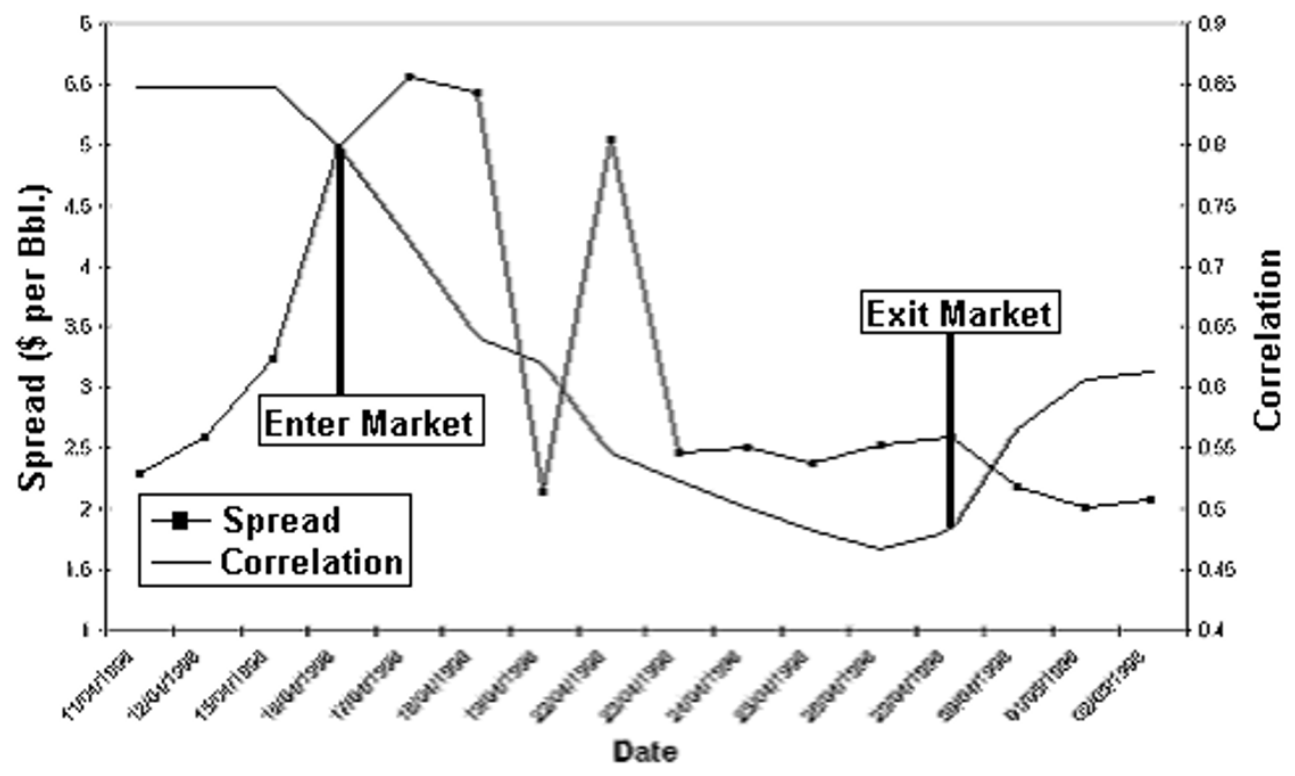

correlation is below $X . X$ is the filter level. This is then multiplied by the returns series of the trading model.

Using this filter, it should also be possible to filter out sudden moves away from fair value which are generally harder to predict than moves back to fair value. Figure 5 shows the entry and exit points of the filter with $X=0$.

Figure 5 shows that we enter the market the day after the change in correlation, $\Delta C$, is below zero (ie $\Delta C<0$ ), and exit the market the day after the change in correlation is above zero (ie $\Delta C>0$ ). Doing this, one can filter out not only periods when the spread is stagnant, but also the initial move away from fair value, which for a mean-reverting asset is less predictable than the move back to fair value.

There are several optimising parameters which can be used for this type of filter, namely the length of correlation lag, period of correlation change and amount of correlation change. For this study the correlation lag $(Z)$ is set to 30 days, and the period of correlation change $(Y)$ to one day. ${ }^{32}$ The only optimising parameter used was the amount of correlation change. Formally, the correlation filter $X_{c}$ can be written as

If $\Delta C<X_{c}$, then take the decision of the trading rule,

If $\Delta C>X_{c}$, then stay out of the market.

where $\Delta C$ is the change in correlation, and $X_{c}$ is the size of the correlation filter.

\section{RESULTS}

The following section shows the results of the empirical investigation. The filters have 
Table 3: Results of non-linear cointegration

\begin{tabular}{llll} 
& $\rho_{1}$ & $\rho_{2}$ & $\rho \iota=\rho_{2}$ \\
Coefficient & -0.012957 & -0.038508 & - \\
$F$-stat & - & - & 5.1936 \\
$p$-value & 0.0021 & 0.0002 & 0.0228 \\
\hline
\end{tabular}

been optimised in sample in order to maximise the Calmar ratio, defined by Jones and Baehr ${ }^{33}$ as

$$
\text { Calmar Ratio }=\frac{\text { Return }}{|\operatorname{Max} D D|}
$$

where Return is the annualised return of trading model, and MaxDD is the maximum drawdown of the trading model defined as

$$
\begin{aligned}
& \text { Maximum drawdown }= \\
& \operatorname{Min}\left[\Delta S_{t}-\operatorname{Max}\left(\sum_{t=1}^{n} \Delta S_{t}\right)\right]
\end{aligned}
$$

Equation (7) is given a high priority, as futures are naturally leveraged instruments. This statistic gives a good measure of the amount of return that can be expected for the amount of investment capital needed to finance a strategy. Furthermore, unlike the Sharpe ratio, which assumes that large losses and large gains are equally undesirable, the Calmar ratio defines risk as the maximum likely loss and is therefore a more realistic measure of risk-adjusted return.

\section{Fair value non-linear cointegration}

The cointegration vector of the in-sample period is shown below

$$
\begin{aligned}
\mu_{t}= & (0.826234 * G A S)-W T I \\
& +(0.000116 * \text { trend })
\end{aligned}
$$

where trend is a linear trend included to account for any inflationary effects on the spread. Equation (8) was estimated using the Johansen method as detailed in Johansen. ${ }^{29}$

The results of the regression of equation (4) are shown in Table 3, columns 2 and 3, the result of the Wald test for $\rho_{1}=\rho_{2}$ is shown in Table 3, column 4.

The result shows that the two coefficients are significant in the estimation of equation (4). They are also significantly different at the 5 per cent level (shown by the Wald test results). This confirms the presence of non-linearity in the cointegration relationship between WTI and GAS. The authors can confirm that, for the in-sample data period, the magnitude of movements of the spread above the cointegration fair value is nearly three times smaller than that of the spread below fair value.

The in-sample and out-of-sample trading results of the non-linear cointegration fair value model are shown in Table 4.

Table 4 shows that all the filters improve the in-sample leverage factor over and above that of the unfiltered model and therefore should be considered as selected over the unfiltered model.

Table 5 shows that only one of the selected filters improves the out-of-sample leverage factor over and above that achieved 
Table 4: Non-linear fair value in-sample trading results ${ }^{a}$

$\begin{array}{lllllc}\text { Filter } & \text { Ann.Ret (\%) } & \text { Ann.StDev (\%) } & \text { MaxDD (\%) } & \text { Calmar } & \text { \#Trades } \\ \text { BTC } & 19.12 & 22.34 & -28.81 & 0.6638 & 3.8163 \\ \text { UnFiltered } & 17.66 & 22.35 & -29.00 & 0.609 & 3.8163 \\ \text { Threshold } & 24.01 & 19.31 & -25.57 & 0.9389 & 12.176 \\ \text { Correl } & 17.82 & 22.10 & -28.83 & 0.6182 & 4.9673 \\ \text { Asymm } & 19.77 & 21.77 & -28.83 & 0.6857 & 8.6019\end{array}$

${ }^{a}$ BTC: results before transactions costs are taken into account, without any filters being employed. All subsequent rows take transactions costs into account.

UnFiltered: results of the unfiltered model.

Threshold: results with the threshold filter applied to the model.

Correl: results with the correlation filter applied to the model.

Asymm: results with the asymmetric filter applied to the model.

Ann.Ret: annualised return of the model.

Ann.StDev.: annualised standard deviation of the model.

MaxDD: maximum drawdown of the model, the maximum loss of the model during the sample period.

Calmar: Calmar ratio. Equation (6), gives a ratio for the amount of return for probable capital input.

\#Trades: average number of trades per year.

Table 5: Non-linear fair value out-of-sample trading results

$\begin{array}{lllllc}\text { Filter } & \text { Ann.Ret }(\%) & \text { Ann.StDev (\%) } & \text { MaxDD (\%) } & \text { Calmar } & \text { \#Trades } \\ & & & & & \\ \text { BTC } & 19.12 & 22.34 & -28.81 & 0.6638 & 3.8163 \\ \text { BTC } & 43.66 & 23.70 & -17.94 & 2.4331 & 8.5175 \\ \text { UnFiltered } & 40.35 & 23.68 & -17.94 & 2.2486 & 8.5175 \\ \text { Threshold } & 38.38 & 22.08 & -16.12 & 2.3814 & 14.979 \\ \text { Correl } & 32.96 & 23.73 & -17.94 & 1.8369 & 10.8671 \\ \text { Asymm } & 37.24 & 23.45 & -17.93 & 2.0768 & 12.042\end{array}$

by the unfiltered model. It should be noted that both the threshold and correlation filters show poorer performance out of sample than the unfiltered model, therefore proving to be erroneous selections.

\section{Multi-layer perceptron network}

The trading results of the MLP model are shown in Table 6: all three filtered models outperform the unfiltered model, and therefore any of the three could potentially 
Table 6: MLP in-sample results

$\begin{array}{lrrrrc}\text { Filter } & \text { Return (\%) } & \text { Stdev (\%) } & \text { MaxDD } & \text { Calmar } & \text { \#Trades } \\ \text { BTC } & & & & & \\ \text { UnFiltered } & 31.62 & 22.61 & -34.86 & 0.907 & 92.51 \\ \text { Threshold } & 13.65 & 22.67 & -47.42 & 0.2878 & 92.51 \\ \text { Correl } & 9.91 & 12.27 & -20.18 & 0.4911 & 53.63 \\ \text { Asymm } & 13.96 & 22.64 & -47.38 & 0.2946 & 94.14 \\ & 6.79 & 7.16 & -8.14 & 0.8344 & 18.8\end{array}$

Table 7: MLP out-of-sample results

\begin{tabular}{lcclll} 
Filter & Return (\%) & Stdev (\%) & MaxDD (\%) & Calmar & \#Trades \\
BTC & 19.91 & 23.66 & -33.70 & 0.5907 & 96.79 \\
UnFiltered & 1.31 & 23.84 & -45.75 & 0.0287 & 96.79 \\
Threshold & 6.32 & 11.68 & -10.98 & 0.5761 & 48.68 \\
Correl & 0.97 & 23.84 & -46.34 & 0.021 & 97.94 \\
Asymm & 2.26 & 7.26 & -6.33 & 0.3574 & 24.05 \\
\hline
\end{tabular}

have been selected.

Table 7 shows that, of the filters selected, the threshold and asymmetric filters improve the out-of-sample Calmar ratio of the model.

\section{Recurrent neural networks}

The trading results of the RNN model are shown in Table 8: all filters could potentially have been chosen, because all filters improve the in-sample performance of the RNN model over and above that achieved by the unfiltered model.

Table 9 shows that the threshold and asymmetric filters would have been bad selections. Although the correlation filter improves the in-sample Calmar ratio, this is not the case out of sample, but it cannot be considered a bad selection.

\section{Higher order neural networks}

The trading results of the HONN model are shown in Table 10: all three filters could have been chosen for the in-sample period, because they all improve on the performance of the unfiltered model.

Table 11 shows that, of the filters potentially selected from the in-sample performance, only the correlation filter proves to be a bad selection.

Overall, the best-performing model before transactions costs is the fair value model, which shows higher out-of-sample returns and therefore a better ability to 
Table 8: RNN in-sample trading results

\begin{tabular}{lrrrrl} 
Filter & Return $(\%)$ & Stdev $(\%)$ & MaxDD $(\%)$ & Calmar & \#Trades \\
& & & & & \\
BTC & 25.88 & 22.64 & -35.54 & 0.7282 & 87.4 \\
UnFiltered & 8.90 & 22.72 & -56.70 & 0.1569 & 87.4 \\
Threshold & 6.76 & 9.95 & -14.80 & 0.4569 & 39 \\
Correl & 9.22 & 22.71 & -54.04 & 0.1706 & 88.1 \\
Asymm & 5.60 & 6.71 & -7.90 & 0.7095 & 21.47 \\
\hline
\end{tabular}

Table 9: RNN out-of-sample trading results

\begin{tabular}{lccccc} 
Filter & Return $(\%)$ & Stdev $(\%)$ & MaxDD $(\%)$ & Calmar & \#Trades \\
BTC & & & & & \\
UnFiltered & 40.73 & 23.56 & -18.41 & 2.2118 & 100.23 \\
Threshold & 21.36 & 23.62 & -19.19 & 1.1128 & 100.23 \\
Correl & 15.92 & 15.16 & -16.20 & 0.9829 & 97.36 \\
Asymm & 21.36 & 23.62 & -19.19 & 1.1128 & 100.23 \\
& 4.93 & 11.97 & -17.82 & 0.2765 & 60.14 \\
\hline
\end{tabular}

Table 10: HONN in-sample trading results

$\begin{array}{lrrrrr}\text { Filter } & \text { Return (\%) } & \text { Stdev (\%) } & \text { MaxDD (\%) } & \text { Calmar } & \text { \#Trades } \\ & & & & & \\ \text { BTC } & 21.43 & 22.66 & -46.22 & 0.4637 & 107.83 \\ \text { UnFiltered } & 0.48 & 22.76 & -60.08 & 0.008 & 107.83 \\ \text { Threshold } & 5.64 & 7.27 & -8.49 & 0.6638 & 9.87 \\ \text { Correl } & 11.48 & 13.85 & -28.11 & 0.4082 & 108.88 \\ \text { Asymm } & 1.97 & 3.11 & -1.67 & 1.1812 & 2.79\end{array}$

predict the direction of the spread.

Further, the results show that, before transactions cost are taken into account, the performance of the NN models in predicting the direction of the spread lie largely in order of computational time. The one exception is the HONN, which outperforms the MLP both in and out of sample, despite having far shorter computational times.

The results of the filters show improvements in the out-of-sample trading statistics over and above that achieved by the unfiltered model in 6 of the 12 times a 
Table 11: HONN out-of-sample trading results

\begin{tabular}{lccccc} 
Filter & Return (\%) & Stdev (\%) & MaxDD (\%) & Calmar & \#Trades \\
BTC & 37.41 & 23.58 & -21.09 & 1.7738 & 105.38 \\
UnFiltered & 16.93 & 23.70 & -30.70 & 0.5513 & 105.38 \\
Threshold & 7.39 & 5.64 & -0.97 & 7.6097 & 10.88 \\
Correl & 9.22 & 15.42 & -22.50 & 0.4095 & 105.38 \\
Asymm & 2.84 & 4.77 & -1.82 & 1.5596 & 4.58 \\
\hline
\end{tabular}

filter is selected. Contrasting this to the threshold filter for which the same ratio is 3 out of 4 , shows that the threshold filter is the most consistent method of filtering trading models on this spread. The threshold filter improves the out-of-sample Calmar ratio by an average of about 8 times. This is compared to about 3 for the asymmetric filter and about -0.2 for the correlation filter.

\section{CONCLUSIONS}

The results clearly show that the relationship between WTI and GAS is non-linear. This is evidenced by the results of the Wald test, showing $\rho_{1} \neq \rho_{2}$. Further, since $\rho_{1}$ is smaller in absolute terms than $\rho_{2}$, movements below fair value tend, on average, to be larger than movements above fair value. The widely believed theory that refiners are holding onto a large refining margin, as presented by Blum ${ }^{3}$ and Macalister et al., ${ }^{4}$ is proved to be false for the period concerned here. It can be concluded that any asymmetry in the time series is not in favour of oil refiners.

If the aim is to model $\Delta S_{t}$, or the change in the spread, the best model is the fair value model. This is evidence by the largest out-of-sample profits without a filter and before transactions costs are taken into account, indicating a superior ability to predict the sign of $\Delta S_{t}$. It is also worth noting that the HONN outperformed the MLP out of sample, despite shorter computational times and limited variables. The authors feel that HONN should be investigated further in reference to financial markets.

The effect of transactions costs is extremely large on very active models such as the three NN investigated here, resulting in the fair value model producing the best out-of-sample performance after transactions costs. In fact, the level of transactions costs for the fair value model is 3.31 per cent p.a., in comparison with 18.59 per cent for the MLP, 19.37 per cent for the RNN and 20.49 per cent for the HONN (indicating an average of 17.03, 92.45, 99.65 and 105.38 trades per year, respectively).

Of the filters investigated, the best model is the threshold filter, which easily outperforms the asymmetric and correlation filters, proving to be the best out-of-sample 
performer on three of the four models. Furthermore, the threshold filter improves the out-of-sample Calmar ratio by an average factor of about 8 in contrast to about 3 for the threshold and about -0.2 for the correlation filters. The result that this filter is the best filter is surprising, given the clear non-linearity of the time series.

Finally, the best model in terms of out-of-sample Calmar ratio is the HONN with a threshold filter, with a Calmar ratio of 7.6097. Making the choice of a trading model on the basis of the in-sample Calmar ratio, however, would have led to the HONN model with the asymmetric filter: out of sample, this model performs satisfactorily, with a Calmar ratio of just over 1.5.

This paper proves that, contrary to the hypothesis raised by Blum ${ }^{3}$ and Macalister et al., ${ }^{4}$ consumers of unleaded gasoline have been getting a 'fair deal' over the sample period concerned. Further work could include the testing of asymmetric adjustment on other spreads that are reflective of profit margins, such as the soybean crush spread or spark spread.

\section{References and Notes}

1 Abeysinghe, T. (2001) 'Estimation of Direct and Indirect Impact of Oil Price on Growth', Economics Letters, Vol. 73, pp. 147-153.

2 Carlstrom, C. T. and Fuerst, T. S. (2005) 'Oil Prices, Monetary Policy and the Macroeconomy', Federal Reserve Bank of Cleveland: Policy Discussion Paper, Vol. 10 April.

3 Blum, J. (2005) 'Who's Profiting From Spiralling Gas Prices?: Refiners Profit Triple in a Year', The Washington Post, 25th September. Available online at: seattletimes.nwsource.com/html/nationworld/ 2002519083_katgas25.html [accessed 26th October, 2005].
4 Macalister, T., Finch, J. and Pratley, N. (2005) 'Record Shell Profit Spurs Windfall Tax Calls' The Guardian, 4th February. Available online at: www.guardian.co.uk/oil/story/ 0,11319,1405766,00.html [accessed 26th October, 2005].

5 This asymmetry is also evidenced by the skewness $(-0.23)$ and kurtosis (12.37) of the distribution.

6 Evans, B., Dunis, C. L. and Laws, J. (2005)

'Trading Futures Spreads: Applications of Threshold and Correlation Filters', Applied Financial Economics, Vol. 16, pp. 1-12.

7 Engle, R. F. and Granger, C. W. J. (1987)

'Cointegration and Error Correction:

Representation, Estimation and Testing', Econometrica, Vol. 55, pp. 251-276.

8 Enders, W. and Granger, C. (1998) 'Unit-root Tests and Asymmetric Adjustment with an Example Using the Term Structure of Interest Rates', Journal of Business and Economic Statistics, Vol. 19, pp. 166-76.

9 Enders, W. and Dibooglu, S. (2004) 'Long-run Purchasing Power Parity with Asymmetric Adjustment', University of Alabama Working Papers Series.

10 Boucher, C. (2003) 'Testing for Rational Bubbles with Time Varying Risk Premium and Non-Linear Cointegration: Evidence from the US and French Stock Markets', Université Paris-Nord Working Papers.

11 Kaastra, I. and Boyd, M. (1995) 'Designing a Neural Network for Forecasting Financial and Economic Time Series', Neurocomputing, Vol. 10, pp. 215-236.

12 Chen, W., Wagner, L. and Lin, C. H. (1996) 'Forecasting the 30-year US Treasury Bond with a System of Neural Networks', NeuroVe\$t Journal, Jan/Feb.

13 Krishnaswamy, C. R., Gilbert, E. W. and Pashley, M. M. (2000) 'Neural Network Applications in Finance', Financial Practice and Education, Spring/Summer, pp. 75-84.

14 Kryzanowski, L., Galler, M. and Wright, D. W. (1993) 'Using Artificial Neural Networks to Pick Stocks', Financial Analysts Journal, Vol. 49, pp. 21-27.

15 Refenes, A. P., Zapranis, A. and Francis, G. (1995) 'Modelling Stock Returns in the Framework of APT', in Refenes, A. P. (eds), Neural Networks in the Capital Markets, Chichester, John Wiley, pp. 101-125.

16 Bansal, R. and Viswanathan, S. (1993) 'No Arbitrage and Arbitrage Pricing: A New Approach', Journal of Finance, Vol. 48, 
pp. 1231-1262.

17 Zrilli, J. S. (1997) 'Financial Prediction Using Neural Networks', London, International Thompson Computer Press.

18 Elman, J. L. (1990) 'Finding Structure in Time', Cognitive Science, Vol. 14, pp. 179-211.

19 Tenti, P. (1996) 'Forecasting Foreign Exchange Rates Using Recurrent Neural Networks', Applied Artificial Intelligence, Vol. 10, pp. 567-581.

20 Saad, E. W., Prokhorov, D. V. and Wunsch, D. C. (1998) 'Comparative Study Of Stock Trend Prediction Using Time Delay, Recurrent and Probabilistic Neural Networks', Transactions on Neural Networks, Vol. 9, pp. 1456-1470.

21 Giles, L. and Maxwell, T. (1987) 'Learning Invariance and Generalization in High-Order Neural Networks', Applied Optics, Vol. 26, No. 23, pp. 4972-4978.

22 Knowles, A., Hussein, A., Deredy, W., Lisboa, P. and Dunis, C. L. (2005) 'Higher-Order Neural Networks with Bayesian Confidence Measure for Prediction of EUR/USD Exchange Rate', CIBEF Working Papers, www.cibef.com.

23 Zhang, M., Xu, S. and Fulcher, J. (2002)

'Neuron-Adaptive Higher Order Neural-Network Models for Automated Financial Data Modeling', Transactions on Neural Networks, Vol. 13, pp. 188-204.

24 Lindemann, A., Dunis, C. and Lisboa, P. (2004) 'Probability Distributions and Leveraged Strategies: An Application of Gaussian Mixture Model to the Morgan Stanley High Technology 35 Index', Journal of Forecasting, Vol. 23, No. 18, pp. 559-585.
25 Lindemann, A., Dunis, C. and Lisboa, P. (2005) 'Level Estimation, Classification and Probability Distribution Architectures for Trading the EUR/USD Exchange rate', Neural Computing and Applications, Vol. 14, No. 3, pp. 256-271.

26 Butterworth, D. and Holmes, P. (2002) 'Inter-Market Spread Trading: Evidence from UK Index Futures Markets', Applied Financial Economics, Vol. 12, pp. 783-790.

27 The correlation between $\Delta S_{t}$ and $\Delta \mu_{t}$ over the in-sample period is over 96 per cent.

28 Dickey, D. A. and Fuller, W. A. (1979)

'Distribution of the Estimators for Autoregressive Time Series with a Unit Root', Journal of the American Statistical Association, Vol. 74, pp. 427-431.

29 Johansen, S. (1988) 'Statistical Analysis of Cointegration Vectors', Journal of Economic Dynamics and Control, Vol. 12, pp. 231-254.

30 Adam, O., Zarader, J. L. Milgram, M. (1993) 'Identification and Prediction of Non-Linear Models with Recurrent Neural Networks', Proceedings of the International Workshop on Artificial Neural Networks, pp. 531-535.

31 In the case of the fair value model, this position is held until fair value is regained.

32 These parameters seem to have a limited effect when compared with the impact of the amount of correlation change.

33 Jones, M. A. and Baehr, M. (2003) 'Manager Searches and Performance Measurement', in Phillips, K. S. and Surz, P. J. (eds), Hedge Funds Definitive Strategies and Techniques, Hoboken, NJ, John Wiley, pp. 112-138. 